1 Universidade Comunitária da Região de Chapecó

(Unochapecó) - Chapecó (SC), Brasil.

Orcid: https://orcid. org/0000-0001-9724-

8667

luanaschneider@

unochapeco.edu.br

2 Universidade do Minho (Uminho) - Braga,

Portugal.

Orcid: https://orcid.

org/0000-0002-4811-

6753

ruipereira@ese.uminho.pt

3 Universidade Comunitária da Região de Chapecó (Unochapecó) - Chapecó (SC), Brasil.

Orcid: https://orcid

org/0000-0002-2487-

8614

Iferraz@unochapeco.edu.br

\section{A prática baseada em evidência no contexto da Atenção Primária à Saúde}

\author{
Evidence-based practice in the context of Primary Health Care
}

Luana Roberta Schneider ${ }^{1}$, Rui Pedro Gomes Pereira ${ }^{2}$, Lucimare Ferraz ${ }^{\mathbf{3}}$

DOI: $10.1590 / 0103-1104201811804$

RESUMO A prática baseada em evidência agrega o melhor conhecimento científico, com a experiência clínica do profissional e a escolha do paciente, resultando em uma maior resolutividade na assistência em saúde. O objetivo do estudo foi analisar a prática baseada em evidência dos profissionais das equipes com Estratégia Saúde da Família em um município de Santa Catarina. Trata-se de um estudo quantitativo, realizado por meio de questionários com 112 profissionais de saúde. Os resultados apontaram que eles consideram a prática baseada em evidência fundamental, contudo, em suas ações, ela está mais centrada na experiência clínica. Outrossim, não se sentem plenamente capacitados para realizar a busca de evidências científicas, destacando o pouco conhecimento e habilidades em pesquisa. Além disso, ressaltam a alta demanda de atendimentos, escasso domínio de língua estrangeira e falta de apoio da gestão como dificultadores. Conclui-se que os profissionais da atenção primária precisam se aprimorar para o desenvolvimento da prática baseada em evidência, sendo que isso ultrapassa a sua vontade individual, ficando também ao encargo das instituições formadoras e dos serviços de saúde.

PALAVRAS-CHAVE Prática clínica baseada em evidências. Atenção Primária à Saúde. Estratégia Saúde da Família.

ABSTRACT Evidence-based practice brings together the best scientific knowledge with the professional's clinical experience and the patient's choice, resulting in a higher resoluteness in health care. The research was carried out aiming at analyzing the evidence-based practice of professionals from Family Health Strategy teams, in a city in the state of Santa Catarina. It was a quantitative study, developed through questionnaires with 112 health professionals. The results showed that professionals consider evidence-based practice essential; however, such practice is more focused on clinical experience in their actions. Moreover, they do not feel completely qualified to search for scientific evidence, highlighting their little knowledge and abilities on research. In addition, they emphasized that applying evidence-based practice is even more difficult because of high demand for care, lack of foreign language skills and lack of management support. As a conclusion, primary care professionals need some improvement for the development of evidencebased practice, which doesn't only depend on their individual willingness, but it is also a responsibility of educational institutions and health services.

KEYWORDS Evidence-based practice. Primary Health Care. Family Health Strategy. 


\section{Introdução}

A Atenção Primária à Saúde (APS) configura-se como porta de entrada e centro de comunicação das redes de atenção a saúde, fornecendo cuidado no âmbito individual, familiar e coletivo, no decorrer do tempo, para todas as condições de saúde, exceto as muito raras. No Brasil, de acordo com os preceitos do Sistema Único de Saúde, a APS tem a saúde da família como estratégia prioritária para sua organização. $\mathrm{O}$ trabalho desenvolvido pela equipe da Estratégia Saúde da Família (ESF) compreende ações de promoção, prevenção, recuperação, reabilitação e cuidados paliativos; outrossim, o processo de trabalho deve ser preconizado de acordo com os problemas, as demandas e as necessidades de saúde das pessoas e famílias em seu território 1 .

Nessa perspectiva, o papel da equipe da ESF extrapolou a demanda de saúde do usuário e passou a assumir a responsabilidade sanitária por determinada população, e um papel de organizador da demanda local2, exigindo dos profissionais uma maior capacidade de análise, intervenção e autonomia e o estreitamento dos elos entre a compreensão e a execução do trabalho'. Para que os profissionais desenvolvam seu amplo leque de atividades na APS, acompanhando os avanços científicos e tecnológicos, uma abordagem que tem apresentado notoriedade para melhorar a efetividade clínica e apoiar o profissional na tomada de decisão é a Prática Baseada em Evidência (PBE) ${ }^{3}$.

A PBE é definida como uma abordagem que associa a melhor evidência científica com a experiência clínica e a escolha do paciente $^{4}$. Diversos autores enfatizam que a PBE é importante para fundamentar a prática profissional, bem como descrevem que a sua implementação é fundamental para alcançar a eficácia, a confiabilidade e a segurança nas práticas em saúde ${ }^{5-7}$. Além disso, resultados encontrados em revisões sistemáticas mostraram que os profissionais acreditam que a PBE pode melhorar a qualidade dos cuidados e os resultados clínicos dos pacientes, mesmo que tenham sido identificadas várias barreiras para o seu uso ${ }^{\mathbf{8}, 9}$.

Os serviços de saúde têm cada vez mais reconhecido a necessidade de realizar a PBE, entretanto, a sua implementação não envolve apenas os atributos pessoais, mas também fatores relacionados com o contexto da organização, como a cultura, a responsabilidade, a carga de trabalho e os recursos disponíveis ${ }^{7}$. Contudo, os estudos sobre a PBE mostram que a maioria das pesquisas é realizada no âmbito hospitalar. Considerando esses aspectos, realizou-se uma pesquisa com o objetivo de analisar a $\mathrm{PBE}$ dos profissionais das equipes com ESF em um município de Santa Catarina, Brasil.

\section{Métodos}

Trata-se de um estudo de abordagem quantitativa, com delineamento descritivo transversal, realizado em todos os Centros de Saúde com modalidade de ESF, na cidade de Chapecó, referência em saúde para a região oeste do estado de Santa Catarina. Foram incluídos como população do estudo todos os profissionais de saúde de nível superior da equipe básica que atuavam na ESF em 2016. Dessa forma, foram incluídos na pesquisa, 44 médicos, 45 enfermeiros e 30 cirurgiões-dentistas, tanto do sexo feminino quanto do masculino, totalizando 119 participantes. Desse total, foram excluídos os profissionais que estavam em férias, servidores com atuação inferior a seis meses no serviço, tendo em vista o pouco tempo de atividades, e aqueles que após três tentativas de contato não aceitaram participar do estudo. Portanto, ao final da coleta de dados, totalizaram 112 profissionais de ambos os sexos, sendo 42 médicos, 41 enfermeiros e 29 cirurgiões-dentistas.

As informações foram coletadas no respectivo local de trabalho dos profissionais por meio de dois instrumentos. O primeiro 
correspondeu ao Evidence-Based Practice Questionnaire (EBPQ), um questionário autoaplicável que explora o uso da PBE no cotidiano dos profissionais da saúde, validado para a versão portuguesa do Brasil em $2014^{10}$ a partir do instrumento original6. O EBPQ possui 24 itens pontuados em uma escala tipo Likert, e o seu escore é calculado somando os valores das respostas de cada uma das questões, totalizando 168 pontos, com a maior pontuação indicando atitudes mais positivas em relação à PBE. Os escores podem ser avaliados, ainda, por domínios, calculando-se a média aritmética ${ }^{\mathbf{1 0}}$.

Em relação à estrutura, os itens são caracterizados em três dimensões: a primeira, PBE (alfa de Cronbach $\square=0,86$ ), utiliza uma escala que oscila entre 1 (nunca) e 7 (frequentemente), integrando 42 pontos. Pretende-se avaliar com que frequência e em consequência de uma lacuna identificada no conhecimento se procedeu às etapas comumente associadas a uma PBE. A dimensão 2 se caracteriza pelas atitudes relacionadas com a prática baseada em vidência $(\square=0,68)$ e permite avaliar a atitude ante a PBE associadas às práticas profissionais, por meio do posicionamento de proximidade adotado para cada par de questões, em 1 ou 28 pontos. A terceira dimensão do questionário representa os conhecimentos e habilidades associados à PBE $(\square=0,92)$, considerada por meio de uma escala que oscila entre 1 (ruim) e 7 (ótimo) em um somatório 98 pontos.

O segundo instrumento, utilizado na coleta de dados, refere-se ao Roteiro de Questões Auxiliares desenvolvido pelos pesquisadores. Esse roteiro contém 14 questões objetivas. No primeiro bloco, as questões estão relacionadas com as características dos participantes, como sexo, idade, ano de formação da graduação, ter ou não pós-graduação e tempo de atuação na APS. O segundo bloco diz respeito à realização de pesquisas e recursos no ambiente de trabalho, como acesso a computador e internet. No terceiro bloco, as questões buscam identificar os tipos de fontes de informação e a frequência da $\mathrm{PBE}$ na prática assistencial. Por fim, o último bloco relaciona-se com a gestão, domínio de língua estrangeira e a relação ensino-serviço. Para atingir sua versão final, foi desenvolvido um teste piloto, com um público semelhante, em um município próximo, porém este não foi validado pelos autores.

Para análise dos dados, inicialmente, foram digitadas as variáveis investigadas em uma planilha formatada do programa Excel $^{\circledR}$ e transportados para o software Statistical Package for the Social Sciences, versão 22.0. Neste programa, foram realizados cálculos de medidas de posição (média, mínima, máxima e mediana) e de dispersão (desvio padrão). Os testes foram realizados com nível de significância de $5 \%$.

Este estudo seguiu todas as diretrizes da Resolução no $466 / 12$ do Conselho Nacional de Saúde para pesquisas realizadas em seres humanos, obtendo-se a aprovação de Comitê de Ética em Pesquisa sob o parecer de número 1.573.371.

\section{Resultados}

Entre os profissionais, identificou-se que o maior percentual era do sexo feminino, com idade média de 36,54 anos $(\mathrm{DP} \pm 8,98)$ e mediana de 33,5 (23-68), com maior percentual no intervalo de 30-39 anos. O tempo de formação foi, em média, de 11,44 anos (DP $\pm 8,36)$ e com mediana 10 (1-31), já a média do tempo de atuação na APS foi de 9,05 anos. A maior parte dos profissionais possui pós-graduação concentrada em nível de especialização. A tabela 1 apresenta como os profissionais da equipe da ESF consideram os três elementos que compõem a PBE. 
Tabela 1. Frequência com que os profissionais da equipe da ESF consideram os elementos da PBE

\begin{tabular}{lrr}
\hline Variáveis $^{\star}$ & Média \pm DP & Mediana (mín.; máx.) \\
\hline A sua experiência clínica & $6,06 \pm 1,38$ & $7(1 ; 7)$ \\
As evidências científicas & $5,94 \pm 1,3$ & $6(1 ; 7)$ \\
A preferência do paciente & $4,33 \pm 1,59$ & $4(1 ; 7)$ \\
\hline Fonte: Elaboração própria. & & \\
* Respostas apresentadas em uma escala Likert de 1 (nunca) até 7 (frequentemente). DP = desvio padrão; Min. = mínima; Max. = máxima.
\end{tabular}

No roteiro de questões auxiliares, os profissionais demarcaram que suas fontes de informações são basicamente fundamentadas em protocolos (75\%), majoritariamente, disponibilizados pelo Ministério da Saúde. A internet surgiu como a segunda opção (72,3\%), seguida por revistas científicas
(54,5\%) e opinião de colegas (44,6\%).

A tabela 2 discorre sobre as práticas dos profissionais da ESF, considerando a atividade clínica e os cuidados prestados aos usuários, no último ano, bem como demonstra com que frequência executaram às etapas comumente associadas a uma PBE.

Tabela 2. Descrição das etapas para avaliar as práticas dos profissionais da equipe da ESF

\begin{tabular}{lrr}
\hline Variáveis $^{\star}$ & Média \pm DP & Mediana (mín.; máx.) \\
\hline $\begin{array}{l}\text { 1. Com que frequência você tem dúvidas sobre sua prática clíni- } \\
\text { ca/trabalho? }\end{array}$ & $3,58 \pm 1,33$ & $3(1 ; 7)$ \\
$\begin{array}{l}\text { 2. Com que frequência você buscou evidências para responder a } \\
\text { sua dúvida? }\end{array}$ & $5,51 \pm 1,61$ & $6(1 ; 7)$ \\
$\begin{array}{l}\text { 3. Com que frequência você avaliou criticamente toda a literatura } \\
\text { encontrada com base em algum critério estabelecido? }\end{array}$ & $4,34 \pm 1,59$ & $4(1 ; 7)$ \\
$\begin{array}{l}\text { 4. Com que frequência você aliou a evidência encontrada com a } \\
\text { sua experiência clínica? }\end{array}$ & $5,72 \pm 1,24$ & $6(2 ; 7)$ \\
$\begin{array}{l}\text { 5. Com que frequência você avaliou os resultados da sua prática? } \\
\text { 6. Com que frequência você compartilhou esse conhecimento }\end{array}$ & $5,42 \pm 1,39$ & $6(2 ; 7)$ \\
com colegas? & $4,79 \pm 1,66$ & $5(1 ; 7)$ \\
\hline
\end{tabular}

Fonte: Elaboração própria.

*Respostas apresentadas em uma escala Likert de 1 (nunca) até 7 (frequentemente). DP = desvio padrão; Min. = mínima; Max. = máxima.

Observa-se que os participantes demarcaram uma média baixa quanto à frequência de dúvidas sobre a sua prática clínica/ trabalho. Acrescenta-se que assinalaram que quando há uma incerteza na conduta sobre seus casos clínicos, 95,5\% buscam informações com os colegas da equipe, na unidade de saúde, conforme as respostas do roteiro de questões auxiliares. Outro aspecto investigado são as atitudes dos profissionais ante a $\mathrm{PBE}$. Os resultados estão descritos na tabela 3 . 
Tabela 3. Descrição das atitudes ante a PBE dos profissionais da equipe da ESF

\begin{tabular}{|c|c|c|}
\hline Variáveis ${ }^{\star}$ & $\begin{array}{c}\text { Média } \pm \text { DP } \\
\text { Mediana (mín.; máx.) }\end{array}$ & Variáveis ${ }^{\star}$ \\
\hline $\begin{array}{l}\text { Minha carga de trabalho é muito gran- } \\
\text { de para que eu me mantenha atualiza- } \\
\text { do com todas as novas evidências. }\end{array}$ & $\begin{array}{r}\square \square \square \square \square \\
3,91 \pm 1,7 \\
4(1 ; 7)\end{array}$ & $\begin{array}{r}\text { Novas evidências são tão importantes } \\
\text { que eu defino um tempo para isso na } \\
\text { minha agenda de trabalho. }\end{array}$ \\
\hline $\begin{array}{l}\text { Eu me sinto desconfortável quando } \\
\text { minha prática é questionada. }\end{array}$ & $\begin{array}{r}\square \square \square \square \square \square \\
5,5 \pm 1,35 \\
6(1 ; 7)\end{array}$ & $\begin{array}{l}\text { Eu acolho de forma aberta os questio- } \\
\text { namentos sobre a minha prática. }\end{array}$ \\
\hline $\begin{array}{l}\text { Práticas baseadas em evidências são } \\
\text { perda de tempo. }\end{array}$ & $\begin{array}{r}\square \square \square \square \square \mathbf{\square} \\
6,52 \pm 0,76 \\
7(4 ; 7)\end{array}$ & $\begin{array}{r}\text { Práticas baseadas em evidências são } \\
\text { fundamentais para a prática profis- } \\
\text { sional. }\end{array}$ \\
\hline $\begin{array}{l}\text { Eu mantenho o uso de métodos tes- } \\
\text { tados e confiáveis ao invés de mudar } \\
\text { para algo novo. }\end{array}$ & $\begin{array}{r}\square \square \square \mathbf{\square} \square \\
5,02 \pm 1,55 \\
5(1 ; 7)\end{array}$ & $\begin{array}{l}\text { Minha prática tem mudado em função } \\
\text { das evidências que tenho encontrado. }\end{array}$ \\
\hline
\end{tabular}

Fonte: Elaboração própria.

*Respostas apresentadas em uma escala Likert de 1 (nunca) até 7 (frequentemente). DP = desvio padrão; Min. = mínima; Max. = máxima.

Os conhecimentos e habilidades dos profissionais para o desenvolvimento da PBE estão apresentados na tabela 4.

Tabela 4. Conhecimentos e habilidades para a PBE dos profissionais da equipe da ESF

\begin{tabular}{lrr}
\hline Variáveis & Média \pm DP & Mediana (mín.; máx.) \\
\hline Conhecimentos para: & & $5(1 ; 7)$ \\
\hline 1. Identificar os principais tipos e fontes de informação existentes & $4,64 \pm 1,15$ & $4(1 ; 7)$ \\
2. Levantar evidências & $4,24 \pm 1,34$ & $5(1 ; 7)$ \\
\hline Habilidades para: & $5,37 \pm 1,04$ & $5(2 ; 7)$ \\
\hline 1. Rever a sua própria prática & $5,07 \pm 1,04$ & $5(1 ; 7)$ \\
2. Aplicar o conhecimento a casos individuais & $4,91 \pm 1,46$ & $5(1 ; 7)$ \\
3. Compartilhar suas ideias e conhecimento com os colegas de trabalho & $4,91 \pm 1,32$ & $5(2 ; 7)$ \\
4. Em informática & $4,89 \pm 1,15$ & $5(1 ; 7)$ \\
5. Determinar quão aplicável clinicamente é o material & $4,87 \pm 1,13$ & $5(1 ; 7)$ \\
6. Identificar lacunas na prática profissional & $4,82 \pm 1,4$ & $5(1 ; 7)$ \\
7. Disseminar novas ideias sobre cuidado entre os colegas & $4,68 \pm 1,3$ & $5(3 ; 6)$ \\
8. Determinar quão válido é o material & $4,66 \pm 0,79$ & $5(1 ; 7)$ \\
9. Em pesquisa & $4,57 \pm 1,26$ & $4(1 ; 7)$ \\
10. Analisar criticamente as evidências ante os padrões já estabelecidos & $4,34 \pm 1,18$ & \\
11.Converter suas necessidades de conhecimento em uma questão de & & \\
pesquisa & & \\
\hline
\end{tabular}

Fonte: Elaboração própria.

*Respostas apresentadas em uma escala Likert de 1 (nunca) até 7 (frequentemente). DP = desvio padrão; Min. = mínima; Max. = máxima 
Ainda sobre o aspecto de conhecimentos e habilidades, destaca-se que os participantes têm dificuldade no domínio de língua estrangeira, sobretudo no idioma inglês. A tabela 5 exemplifica os resultados.

Tabela 5. Domínio de leitura e compreensão de língua estrangeira (inglês e espanhol) dos profissionais da equipe da ESF

\begin{tabular}{|c|c|c|c|c|}
\hline Variável & $\begin{array}{c}\text { Medicina } \\
(n=42) \\
n(\%)\end{array}$ & $\begin{array}{l}\text { Enfermagem } \\
\begin{array}{c}(n=41) \\
n(\%)\end{array}\end{array}$ & $\begin{array}{l}\text { Odontologia } \\
\begin{array}{c}(n=29) \\
n(\%)\end{array}\end{array}$ & $\mathrm{p}$ \\
\hline Língua inglesa (leitura) & & & & $<0,001$ \\
\hline Pouco & $5(11,9)$ & $28(68,3)$ & $10(34,5)$ & \\
\hline Razoavelmente & $14(33,3)$ & $10(24,4)$ & $10(34,5)$ & \\
\hline Bem & $23(54,8)$ & $3(7,3)$ & $9(31)$ & \\
\hline Língua inglesa (compreensão) & & & & $<0,001$ \\
\hline Pouco & $4(9,5)$ & $25(61)$ & $8(27,6)$ & \\
\hline Razoavelmente & $20(47,6)$ & $12(29,3)$ & $12(41,4)$ & \\
\hline Bem & $18(42,9)$ & $4(9,8)$ & $9(31)$ & \\
\hline Língua espanhola (leitura) & & & & $<0,001$ \\
\hline Pouco & $10(23,8)$ & $20(48,8)$ & $18(62,1)$ & \\
\hline Razoavelmente & $10(23,8)$ & $18(43,9)$ & $9(31)$ & \\
\hline Bem & $22(52,4)$ & $3(7,3)$ & $2(6,9)$ & \\
\hline Língua espanhola (compreensão) & & & & $<0,001$ \\
\hline Pouco & $11(26,2)$ & $22(53,7)$ & $14(48,3)$ & \\
\hline Razoavelmente & $14(33,3)$ & $16(39)$ & $13(44,8)$ & \\
\hline Bem & $17(40,5)$ & $3(7,3)$ & $2(6,9)$ & \\
\hline
\end{tabular}

Fonte: Elaboração própria.

Ainda de acordo com as respostas do roteiro de questões auxiliares, todos assumiram ter acesso a computador com internet em seu local de trabalho, e $36,6 \%$ demarcaram que desenvolveram alguma pesquisa no centro de saúde (levantamento de dados, diagnóstico comunitário, pesquisa de campo etc.) nos últimos três anos. No que concerne ao incentivo dos gestores para momentos de estudo durante a jornada de trabalho, obteve-se em uma média de $3,45 \pm 1,94$, em uma escala que variou de 1 (nunca) até 7 (frequentemente).

Por meio do roteiro de questões auxiliares, indagou-se se isso ocorria, acreditando que ter vínculo com uma universidade incentiva e estimula os profissionais pela busca de uma PBE. Verificou-se que 38,4\% dos participantes têm contato com pessoas de alguma universidade. Desse total, 33\% correspondiam a vínculo com os acadêmicos; e 23,2\%, com professores.

\section{Discussão}

O estudo atual mostrou que os profissionais das equipes da ESF consideram a PBE importante para suas tomadas de decisões, sendo fundamental para o seu exercício 
profissional. Resultados condizentes foram encontrados em revisões sistemáticas, em que os profissionais acreditam que a $\mathrm{PBE}$ pode melhorar a qualidade dos cuidados e os resultados clínicos dos pacientes ${ }^{8,9}$. Em Portugal, uma pesquisa similar revelou que os profissionais de saúde demonstraram a convicção de que é positivo e válido apoiar as práticas com base em pesquisas, acreditando que esse fato trará um melhor desenvolvimento para o seu futuro profissional'1 .

Apesar do reconhecimento acerca da necessidade e importância de realizar a PBE, contudo, os resultados sustentam que os profissionais desenvolvem a PBE mais comumente pela vertente da experiência clínica, em suas práticas assistenciais. De maneira convergente, demais estudos apontam que a opinião dos colegas surge como o meio mais utilizado como fontes de informação para a prática, ao inverso da consulta de artigos em revistas científicas ${ }^{12-14}$.

Destaca-se que a troca de experiências com o colega é um processo de aprendizagem que propicia a construção de conhecimentos a partir dos saberes prévios das pessoas, em consonância com os problemas vivenciados no trabalho, denominado de aprendizagem significativa ${ }^{15}$. Em suma, é inegável que experiência e a troca de saberes são importantes nas ações rotineiras da prática clínica dos profissionais da ESF. No entanto, deve-se considerar que o trabalho não deve ser baseado somente no empirismo e/ou na intuição. Tendo em vista que as práticas em saúde não são estanques, e, sim, mudam com os avanços científicos, sociais e culturais, entende-se que prestar uma assistência norteada em conclusões advindas de métodos científicos consistentes resulta em um preceito no cuidado de saúde ${ }^{16}$.

Outro aspecto revelado neste estudo, acerca dos elementos da PBE, é de que a preferência do paciente é considerada, predominantemente, quando o usuário chega ao serviço com algum tipo de informação sobre o seu estado de saúde. Essa informação merece destaque, visto que autores argumentam que a comunicação entre o profissional e o paciente é a base do cuidado terapêutico, e estimular que o indivíduo participe das decisões sobre o próprio tratamento é uma maneira de o profissional de saúde respeitá-lo na sua integralidade $^{17}$. Além disso, respeitar as preferências dos pacientes é valorizar o conhecimento que estes trazem para a interação 'profissional-paciente', baseado em bons diálogos, tornando os resultados mais efetivos ${ }^{\mathbf{1 8}}$.

$O$ elemento da PBE relacionado com as evidências científicas, de acordo com a demarcação dos profissionais, ficou considerado em segundo lugar na frequência da prática assistencial. Ademais, apesar de os profissionais se sentirem aptos a buscar evidências científicas, ficou demonstrado que quando buscam evidências, estas são basicamente dos protocolos disponibilizados pelo Ministério da Saúde. Essa atitude justifica-se na questão em que o Ministério da Saúde disponibiliza os materiais para nortear as ações da ESF. Todavia, ressalta-se que, mesmo que haja um grau forte de recomendação para uma determinada conduta, é imprescindível analisar sua adequação para uma realidade específica e, ainda, é necessário ter cautela em adotar recomendações da literatura para o contexto local, principalmente, quando as realidades socioeconômicas e culturais são muito diferentes ${ }^{19}$. Por esse motivo, há uma necessidade de serem abordados em uma lógica de normalização, e nunca de uniformização de práticas e/ou cuidados. Uma norma de orientação clínica ou guideline deverá ser sempre passível de adaptação a um contexto/ realidade local ${ }^{20}$.

$\mathrm{O}$ alto uso dos protocolos pode estar relacionado com as dificuldades em identificar as fontes de informação, formular uma questão de pesquisa, analisar criticamente as evidências e a própria dificuldade em fazer pesquisa. Além disso, ressalta-se que, nos instrumentos de coleta de dados, os profissionais pontuaram acima da média em todas as questões no âmbito dos conhecimentos e habilidades para a PBE. 
O deficit de conhecimentos e habilidades para uma PBE já foi mencionado por outros autores $^{\mathbf{3} 21,22}$. Os profissionais têm uma baixa capacidade de realizar pesquisas e aplicá-las na prática, uma vez que demostraram uma baixa frequência na atitude de formular perguntas de pesquisa, incapacidade de entender os termos estatísticos utilizados e avaliar criticamente a literatura ${ }^{23-25}$.

Em outra investigação, os participantes apontaram que estariam mais confiantes se houvesse uma pessoa com experiência em pesquisa que lhes simplificasse a informação relevante e, ainda, que seria conveniente realizar uma formação específica para auxiliar na utilização efetiva dos estudos"1․ Na China, uma investigação sobre as atitudes e conhecimentos para a PBE apontou que o item mais negativo foi para habilidades de pesquisa ${ }^{26}$.

Sobre esse aspecto, um estudo qualitativo no âmbito da atenção primária na Espanha analisou a percepção dos enfermeiros em relação aos conhecimentos, vantagens e barreiras no processo de aplicação da PBE e destacou a lacuna existente na tradução de conhecimento para a prática. Além disso, expõe que seria aconselhável desenvolver estratégias de capacitação para facilitar a implementação da PBE, adaptada ao ambiente da APS ${ }^{23}$.

Além de poucos conhecimentos e habilidades para desenvolver pesquisas, a alta demanda da ESF, proveniente de cuidado assistencial; pouco domínio de língua estrangeira e falta de apoio da gestão foram citados como entraves para operacionalizar a PBE no cenário de estudo.

A falta de tempo suficiente no trabalho para implementar novas ideias também foi o item mais pontuado em investigações realizadas no cenário brasileiro ${ }^{3,27}$. No Texas (EUA), os resultados apontam para a mesma direção, revelando que, embora os profissionais dispusessem de uma variedade de recursos para localizar pesquisas e implementar a PBE, os participantes demarcaram muitas barreiras, como a definição de tempo para incorporação de evidências durante o trabalho ${ }^{12}$.
A alta demanda de atividades assistenciais e burocráticas, somada à falta de tempo destinado para a educação em serviço e discussão de casos clínicos, relaciona-se com o baixo uso da PBE pelos profissionais da APS ${ }^{28,29}$. Os profissionais, em geral, percebem a importância da PBE e aceitam a necessidade de implementá-la, mas o pouco tempo no trabalho, para ler artigos de pesquisa, pode desestimular o seu uso ${ }^{26}$.

Já as barreiras linguísticas são consideradas obstáculos significativos na busca de evidências para a prática assistencial, e o conhecimento da língua inglesa é crucial para manter-se atualizado $^{\mathbf{1 3}}$, haja vista que as evidências na área da saúde são produzidas e publicadas, majoritariamente, na língua inglesa ${ }^{30}$.

Em relação à barreira da falta de apoio da gestão para momentos de estudo, durante a jornada de trabalho, pondera-se que, embora não seja suficiente para mudar a prática, uma cultura de aceitação e apoio de gestores e colegas pode ser considerada como um elemento facilitador para o fortalecimento da PBE nos serviços de saúde ${ }^{\mathbf{1 4}}$.

Apesar das limitações, contudo, há aspectos que facilitam uma PBE; citam-se, neste estudo, o acesso a computador e internet e a integração ensino-serviço com a universidade. $\mathrm{O}$ acesso a recursos de informática é uma situação favorável importante, pois facilita a pesquisa científica"1, entretanto, quando subutilizada, deixa de se constituir em um elemento significativo para o planejamento e avaliação das ações de saúde ${ }^{31}$.

A integração ensino-serviço com a universidade, impulsionada por meio de acadêmicos, durante a supervisão de estágios ou desenvolvimento do trabalho de conclusão de curso, constitui-se em um elemento favorável para a $\mathrm{PBE}$, no entanto, essa integração deve funcionar como via de mão dupla para o intercâmbio de conhecimento, na qual ambos são potenciais parceiros na apropriação e reprodução das ações ${ }^{32}$. Pesquisa conduzida na região sul do Brasil identificou que os profissionais da APS mostraram que 
as ações da integração ensino-serviço representam desafio, crescimento e sobrecarga de trabalho; enquanto para os acadêmicos elas significam aproximação com a realidade, postura crítica-reflexiva e visão ampliada de saúde ${ }^{33}$.

Como estratégia para superar a lacuna entre a academia e a prática clínica, recomenda-se o desenvolvimento de equipes colaborativas e interinstitucionais envolvendo pesquisadores, docentes, administradores e profissionais clínicos. Essas parcerias cooperam para o desenvolvimento da PBE incentivando profissionais a se engajarem no processo de pesquisa e busca da evidência, propiciando a tradução do conhecimento ${ }^{34}$.

Compreende-se que a educação dos profissionais de saúde é um processo contínuo e intermediado pelas relações entre instituições de ensino e os serviços de saúde ${ }^{35}$ e que os programas de ensino podem aumentar os comportamentos que apoiam a $\mathrm{PBE}^{\mathbf{2 1}}$.

\section{Conclusões}

Os profissionais que atuam na APS consideram as PBE fundamentais para as ações que são desenvolvidas na ESF, contudo a experiência clínica - que muitas vezes advém do compartilhamento de vivências entre os colegas - foi o principal elemento das práticas em saúde, seguida por evidências científicas e a preferência do paciente. Identifica neste estudo que a falta de habilidade na busca de como levantar evidências, pouco domínio de língua estrangeira, a alta demanda de cuidado assistencial na ESF e a falta de apoio da gestão municipal são barreiras para a implementação da PBE nos serviços de saúde.

Diante do exposto, entende-se que o desenvolvimento da PBE na APS depende das atitudes dos profissionais, da organização e dos processos de trabalho da equipe. Assim, aprimorar as ações em saúde baseadas em evidências dos profissionais implica que estes realizem a PBE já no âmbito da graduação. Além disso, os serviços de saúde devem incentivar a produção e o consumo de evidências científicas, que podem se dar por meio de parcerias com as instituições de ensino e pesquisa. Igualmente, deve haver um suporte organizacional, ou seja, um ambiente de trabalho que propicie e priorize espaços para momentos de trocas de conhecimentos entre os profissionais, fortalecendo o elemento da experiência clínica. Do mesmo modo, deve haver uma escuta ativa dos profissionais para que estes identifiquem e dialoguem com as preferências do paciente para uma tomada de decisão compartilhada.

Por fim, sugere-se o desenvolvimento de estudos com abordagem qualitativa, com o intuito de ampliar a análise das informações e, dessa forma, obter uma maior compreensão da PBE pelos profissionais da saúde no contexto da atenção primária, sobretudo, na ESF. 


\section{Referências}

1. Brasil. Ministério da Saúde. Portaria n ${ }^{\circ} 2.436$ de 21 de setembro de 2017. Diário Oficial da União. 21 set 2017 [acesso em 2018 jan 4]. Disponível em: http:// www.foa.unesp.br/home/pos/ppgops/portaria-n-2436.pdf.

2. Ribeiro CDM, Gouvêa MV, Casotti E. Problemas éticos e justiça social na Estratégia Saúde da Família. Rev. Bioét. [internet]. 2017 [acesso em $2017 \mathrm{dez}$ 19]; 25(2):348-357. Disponível em: http://dx.doi. org/10.1590/1983-80422017252195.

3. Camargo FC, Iwamoto HH, Monteiro DAT, et al. Avaliação de intervenção para difusão da enfermagem baseada em evidências em hospital de ensino. Rev. Gaúcha Enferm. [internet]. 2016 [acesso em 2017 dez 12]; 37(esp):e68962:1-9. Disponível em: http://dx.doi.org/10.1590/1983-1447.2016. esp.68962.

4. Saunders H, Vehvilainen-Julkunen K. Nurses' Evidence-Based Practice Beliefs and the Role of Evidence-Based Practice Mentors at University Hospitals in Finland. Worldviews on Evidence-Based Nursing. [internet]. 2017 [acesso em $2017 \mathrm{dez}$ 18]; 14(1):35-45. Disponível em: https://sigmapubs.onlinelibrary.wiley.com/doi/abs/10.1111/wvn.12189.

5. Sackett DL, Rosenberg WM, Gray JA, et al. Evidence based medicine: what it is and what it isn't. Br Med J. [internet]. 1996 [acesso em 2017 dez 10]; 312(7023):71-72. Disponível em: https://doi. org/10.1136/bmj.312.7023.71.

6. Upton D, Upton P. Development of Evidence-Based Practice Questionnaire for nurses. J Adv Nurs. [internet]. 2006 [acesso em $2017 \mathrm{dez}$ 18]; 53(4):454458. Disponível em: https://onlinelibrary.wiley. com/doi/abs/10.1111/j.1365-2648.2006.03739.x.

7. Barría RM. Implementing Evidence-Based Practice: A challenge for the nursing practice. Invest Educ Enferm. 2014; 32(2):191-193. Disponível em: http://www.scielo.org.co/scielo.php?script=sci_artt ext\&pid=S0120-53072014000200001.
8. Patelarou AE, Patelarou E, Brokalaki H, et al. Current evidence on the attitudes, knowledge and perceptions of nurses regarding evidence-based practice implementation in European community settings: a systematic review. J Community Health Nurs. [internet]. 2013 [acesso em 2017 dez 01]; 30(4):230-244. Disponível em: https://www.tandfonline.com/doi/abs/10.1080 /07370016.2013.838501.

9. Saunders H, Vehvilainen-Julkunen K. The state of readiness for evidence-based practice among nurses: An integrative review. Int J Nurs Stud. [internet]. 2016 [acesso em $2017 \mathrm{dez}$ 12]; 56:128-140. Disponível em: https://www.ncbi.nlm.nih.gov/pubmed/26603729.

10. Rospendowiski K, Alexandre NMC, Cornelio ME. Adaptação cultural para o Brasil e desempenho psicométrico do "Evidence-Based Practice Questionnaire”. Acta Paul. Enferm. [internet]. 2014 [acesso em 2017 dez 11]; 27(5):405-411. Disponível em: http://dx.doi.org/10.1590/1982-0194201400068.

11. Peixoto MJ, Pereira RPG, Martins A, et al. Enfermagem baseada em evidência: atitudes, barreiras e práticas entre contextos de cuidados. Porto: ESEP; 2017.

12. Yoder LH, Kirkley D, McFall DC, et al. Staff Nurses' Use of Research to Facilitate Evidence-Based Practice. Am J Nurs. [internet]. 2014 [acesso em 2017 dez 12]; 114(9):26-37. Disponível em: https://www. ncbi.nlm.nih.gov/pubmed/25121949.

13. Lemos S, Morita MC, Carloto E. Recursos utilizados por graduandos de odontologia e Cirurgiões-Dentistas do SUS para estudar e resolver dúvidas surgidas durante o trabalho. Rev ABENO [internet]. 2014 [acesso em 2017 dez 14]; 14(1):3-16. Disponível em: https://revabeno.emnuvens.com.br/revabeno/article/view/107.

14. Baird L, Miller T. Factors influencing evidence-based practice for community nurses. Br J. Community Nurs [internet]. 2015 [acesso em 2017 
dez 12]; 20(5):233-242. Disponível em: https:// www.magonlinelibrary.com/doi/abs/10.12968/ bjcn.2015.20.5.233.

15. Brasil. Ministério da Saúde. Gabinete do Ministro. Portaria $n^{\circ} 278$, de 27 de fevereiro de 2014. Institui diretrizes para implementação da Política de Educação Permanente em Saúde, no âmbito do Ministério da Saúde. Diário Oficial da União. 27 fev 2014.

16. Edward K, Mills C. A Hospital Nursing Research Enhancement Model. J Contin Educ Nurs [internet]. 2013 [acesso em $2017 \mathrm{dez}$ 12]; 44(10):447-454. Disponível em: https://www.ncbi.nlm.nih.gov/pubmed/23937071.

17. Silva MJP. Comunicação e o resgate do ser: o papel da comunicação na humanização da atenção à saúde. In: Conferência proferida no IV Congresso de Humanização. Curitiba: PUC-PR; 2013.

18. Baião BS, Oliveira RA, Lima VVPC, et al. Acolhimento humanizado em um posto de saúde urbano do Distrito Federal, Brasil. Rev. APS [internet]. 2014 [acesso em 2018 jan 6]; 17(3):291-302. Disponível em: https://aps.ufjf.emnuvens.com.br/aps/article/ view/1870/820.

19. Duncan BB, Schmidt MI, Falavigna M. Prática da Medicina Ambulatorial Baseada em Evidências. In: Duncan BB, organizador. Medicina ambulatorial: condutas de atenção primária baseadas em evidências. 4. ed. Porto Alegre: Artmed; 2013. p. 44-66.

20. Agree Next Steps Consortium. The AGREE II Instrument [internet]. Canadá: AGREE; c2010-2014 [acesso em 2018 jan 6]. Disponível em: http://www. agreetrust.org.

21. Weng YH, Kuo KN, Yang CY, et al. Implementation of evidence-based practice across medical, nursing, pharmacological and allied healthcare professionals: a questionnaire survey in nationwide hospital settings. Implement Sci. [internet]. 2013 [acesso em 2017 dez 20]; 8(112):1-10. Disponível em: https:// implementationscience.biomedcentral.com/articles/10.1186/1748-5908-8-112.
22. Shin J, Lee E. The Influence of Social Capital on Nurse-Perceived Evidence-Based Practice Implementation in South Korea. J Nurs Scholarsh. [internet]. 2017 [acesso em 2018 jan 20]; 49(3):267-276. Disponível em: https://www.ncbi.nlm.nih.gov/pubmed/28253429.

23. Pericas-Beltran J, Gonzalez-Torrente S, Pedro-Gomez J, et al. Perception of Spanish primary healthcare nurses about evidence-based clinical practice: a qualitative study. Int Nurs Rev [internet]. 2014 mar [acesso em 2017 dez 10]; 61(1):90-98. Disponível em: https://www.ncbi.nlm.nih.gov/pubmed/24467293.

24. Shafiei E, Baratimarnani A, Goharinezhad S, et al. Nurses' perceptions of evidence-based practice: a quantitative study at a teaching hospital in Iran. Med J Islam Repub Iran [internet]. 2014 [acesso em 2018 jan 19]; 28(135):1-7. Disponível em: https://www.ncbi.nlm.nih.gov/pmc/articles/ PMC4322335/pdf/MJIRI-28-135.pdf.

25. Mohsen MM, Safaan NA, Okby OM. Nurses' Perceptions and Barriers for Adoption of Evidence Based Practice in Primary Care: Bridging the Gap. Am J Nurs Research [internet]. 2016 [acesso em 2018 jan 30]; 4(2):25-33. Disponível em: http://pubs.sciepub.com/ajnr/4/2/1/index.html.

26. Zhou F, Hao Y, Guo H, et al. Attitude, Knowledge, and Practice on Evidence-Based Nursing among Registered Nurses in Traditional Chinese Medicine Hospitals: A Multiple Center Cross-Sectional Survey in China. Evid Based Complement Alternat Med [internet]. 2016 [acesso em 2017 dez 7]. Disponível em: http://dx.doi.org/10.1155/2016/5478086.

27. Ferreira MBG, Haas VJ, Dantas RAS, et al. Adaptação cultural e validação de um instrumento sobre barreiras para o uso dos resultados da pesquisa. Rev. Latino-Am. Enfermagem [internet]. 2017 [acesso em $2017 \mathrm{dez} 20$ ]; 25:e2852. Disponível em: http://www.scielo.br/scielo.php?script=sci_arttext \&pid=S0104-11692017000100313. 
28. Mallion J, Brooke J. Community- and hospital-based nurses' implementation of evidence-based practice: are there any differences?. Br J Community Nurs [internet]. 2016 [acesso em $2017 \mathrm{dez}$ 19]; 21(3):148-154. Disponível em: www.magonlinelibrary.com/doi/pdf/10.12968/bjcn.2016.21.3.148.

29. Atkinson LZ, Forrest A, Marriner L, et al. Implementing tools to support evidence-based practice: a survey and brief intervention study of the National Elf Service across Oxford Health NHS Foundation Trust. Evid Based Ment Saúde [internet]. 2017 [acesso em 2017 dez 20]; 20(2):41-45. Disponível em: https://ebmh.bmj.com/content/early/2017/03/31/eb-2017-102665.citation-tools.

30. Brasil. Ministério da Saúde. Secretaria de Ciência, Tecnologia e Insumos Estratégicos. Departamento de Ciência e Tecnologia. Síntese de Evidências para Políticas de Saúde: estimulando o uso de evidências científicas na tomada de decisão. 1. ed. Brasília, DF: Ministério da Saúde; 2015.

31. Gonçalves L, Fialek S, Castro T, et al. Experiência de enfermeiros com computadores na atenção primária: estudo exploratório. Cogitare Enferm [internet]. 2016 [acesso em $2017 \mathrm{dez}$ 12]; 21(1):1-11. Disponível em: http://revistas.ufpr.br/cogitare/article/ view/43243/27707.

32. Albiero JFG, Freitas SFT. Modelo para avaliação da integração ensino-serviço em Unidades Docen- tes Assistenciais na Atenção Básica. Saúde debate [internet]. 2017 [acesso em 2018 fev 7]; 41(114):753767. Disponível em: http://dx.doi.org/10.1590/01031104201711407.

33. Gonçalves CNS, Corrêa AB, Simon G, et al. Integração ensino-serviço na voz de profissionais de saúde. Rev enferm UFPE online [internet]. 2014 [acesso em $2017 \mathrm{dez}$ 18]; 8(6):1678-1686. Disponível em: file://D:/Revista/Downloads/13641-35038-1PB\%20(1).pdf.

34. Debruyn R, Ochoa-Marin SC, Semenic S. Barriers and Facilitators to Evidence-Based Nursing in Colombia: Perspectives of Nurse Educators, Nurse Researchers and Graduate Students. Invest. Educ. Enferm [internet]. 2014 [acesso em 2018 jul 2]; 32(1):9-21. Disponível em: http://www.scielo.org. co/pdf/iee/v32nl/v32nla02.pdf.

35. Vendruscolo C, Prado ML, Kleba ME. Integração Ensino-Serviço no âmbito do Programa Nacional de Reorientação da Formação Profissional em Saúde. Ciênc. Saúde Colet [internet]. 2016 [acesso em $2017 \mathrm{dez}$ 20]; 21(9):2949-2960. Disponível em: http://www.scielo.br/scielo.php?pid=S1413$-81232016000902949 \&$ script $=$ sci_abstract.

Recebido em 08/02/2018

Aprovado em 08/07/2018

Conflito de interesses: inexistente

Suporte financeiro: não houve 